\title{
A NOVEL APPROACH TO DETECT FISSURES IN LUNG CT IMAGES USING MARKER-BASED WATERSHED TRANSFORMATION
}

\author{
${ }^{1}$ Devaki, K. and ${ }^{2}$ V. Murali Bhaskaran \\ ${ }^{1}$ Department of Computer Science, Paavai College of Engineering, Tamilnadu, India \\ ${ }^{2}$ Department of Computer Science, Dhirajlal Gandhi College of Technology, Tamilnadu, India
}

Received 2013-08-19; Revised 2013-12-11; Accepted 2014-01-25

\begin{abstract}
Detection and segmentation of fissures is useful in the clinical interpretation of CT lung images to diagnose the presence of pathologies in the human lungs. A new automated method based on marker-based watershed transformation has been proposed to segment the fissures considering its unique structure as a long connected component. Marker based watershed transformation is applied and morphological operations are employed to specify the internal and external markers. The smaller regions in the resulting image are removed by a novel procedure called Small Segment Removal Algorithm (SSRA) to segment the fissures alone. The performance of the method is validated by experimenting with 6 CT image sets. An expert radiologist observation is used as reference to assess the performance. A promising accuracy of $96.61 \%$ is shown with the rms error in the range of $0.877 \pm 0.224 \mathrm{~mm}$ for the left oblique fissure and $0.803 \pm 0.262 \mathrm{~mm}$ for the right oblique fissure.
\end{abstract}

Keywords: Lung CT Image, Segmentation, Detection, Fissures, Marker Based Watershed

\section{INTRODUCTION}

Pulmonary diseases are the leading causes of death worldwide. Computed Tomography is the mostly adopted imaging technique to diagnose and detect the pathologies present in the lungs. Interpretation and diagnosis from the stack of sequential images of the CT is a difficult task for the doctors. Automation of the segmentation of the lung anatomical parts and detection of abnormalities present in the lungs, is therefore, necessary to enable the doctors to detect the various chronic obstructive pulmonary diseases and for early prognosis.

Fissures are the separating boundaries between the lobes of the lungs. A human body consists of right and left lungs with the hilar region separating them. The left lung is divided into upper and lower lobes by the left oblique or major fissure. The right lung has three compartments namely upper, middle and lower lobes. The right oblique or major fissure divides the middle lobe from the lower lobe. Horizontal or minor fissure separates the upper and middle lobes.

The lobar fissures are low contrast surfaces with blurred boundaries when viewed on cross sectional CT images (Zhang et al., 2006). On CT images of 1-3 mm thickness, the fissures can often be observed as a thin curve with high attenuation values compared to the surrounding tissues (Jingbin et al., 2006).

Automation of fissure segmentation is essential to assist the doctors in identifying the fissures in the entire stack of CT scan slices. The detection and segmentation of fissures are of clinical importance in the assessment of lung disease on a lobar level. Development of a computer aided diagnosis system helps in the quantitative assessment of bening and malignant nodules present in the lungs. The location of fissures guides as a reference to determine the position of a tumor that moves with respiration and also the spreading of various pulmonary diseases across the lobar areas (Jingbin et al., Corresponding Author: Devaki, K., Department of Computer Science, Paavai College of Engineering, Tamilnadu, India 
2006). A robust detection of pulmonary fissure could provide a basis for accurate lobe segmentation that may help to facilitate preoperative planning and postoperative assessment in the clinical practice.

Automation of fissure detection is a challenging task due to its low contrast, fuzzy appearance in CT images. A fissure has a variety of appearance with varied shapes in the computed tomography images due to the respiratory and cardiac motion and the partial volume effect present. Moreover, fissures are incomplete in a considerable percentage of patient study set. The surrounding structures like airways, vascular branches, nodules and noise artifacts present make the detection process more difficult.

The detection and segmentation of fissures is hence an essential step towards building an automatic CAD system, which remains challenging yet. Hence, this study proposes a novel method to detect and segment the fissures in lung CT images.

The paper is organized as follows. In the following section the works related to our proposed method is discussed. In section 3 the method adopted is explained and the experiments conducted are elaborated in section 4. Section 5 gives the results and section 6 discusses on the outcome. A conclusion is given in section 7 .

\subsection{Related Works}

In recent years many authors have proposed methods to detect and segment the fissures. The methods mainly differ on their dependence on the anatomical knowledge of the fissures. Zhang et al. (2006) proposed a method by constructing anatomical atlases to initialize fissure detection. Fuzzy reasoning was then applied to perform final fissure detection. The method may fail in the case of unusual anatomy or incomplete scans.

Jingbin et al. (2006) method constructed a ridge map to enhance the fissure region. A curve growing process then identified an initial curve segment and completed the fissure construction by growing the curve segment by segment. The method proved to be robust in varying accuracy.

Qiao et al., (2008) developed a method where the fissure angle was fixed initially. Then adaptive fissure sweep was performed to locate the fissure region automatically. As a second step watershed was applied to refine the location and curvature of the fissure. However, the method did not consider the anatomical variations.

Jiantao et al. (2009) adopted geometric modeling of lung volume using marching cubes algorithm. A statistical approach using extended Guassian Image based procedure was applied to extract the fissures as plane patches. The method required no prior knowledge of anatomy and it also accommodated variations among individuals.

An indirect method to segment the fissures was proposed by (Soumik and Reinhardt, 2009). The airways and vessels were segmented as the first step, followed by the distance map calculation of the vasculature structure. This step provide a region of interest of the fissure position, where the ridgeness measure was applied to locate the fissures and a 3-D graph search was done to find the optimal position. The method depends on the accuracy of the airways and vessel segmentation. In case of lungs with disease the accuracy becomes a question.

Rikxoort et al. (2009) developed a method to detect fissures in chest CT scans using pattern recognition technique based supervised enhancement filters. The enhancement filter was trained with a set of voxels sampling and the feature set was calculated. The test data was applied to the filter and the feature set obtained was given to a classifier to classify and enhance the fissures. The method was further improved to give better performance using a multistage supervised filter. The method requires manual intervention to choose the training data and it is also time consuming when the test data set varies.

A similar supervised learning technique was proposed by (Ochs et al., 2007) to classify the lung bronchovascular anatomy using a single feature set and classification method for all the bronchovascular anatomy. Feature set based on attenuation value and eigen of Hessian Matrix are used to train the ensemble classifier based on AdaBoost learning approach.

Lin-Yu and Li-Chin (2010) proposed an automatic method to detect the oblique fissures based on genetic algorithm. The algorithm worked by patching separate fissure pieces on the basis of the fissure function to get the complete curve information of the fissures. The method appears to be direct without any apriori anatomical knowledge.

Ross et al. (2009; 2010) proposed two methods to segment the fissures. The earlier work required manual intervention and thin plate spline interpolation method was applied to complete the fissure segmentation. An automated method was proposed in the later work using eigen values of Hessian matrix and MAP estimation. However, the method did not work for missing or incomplete fissures. 
Gomathi and Thangaraj (2010) proposed Fuzzy Possibility C Mean clustering algorithm to segment the lung images. Support Vector Machine was then applied to classify the cancer nodules and it was shown that the method gave better classification of cancer nodules.

Christ and Parvathi (2011) applied clustering and watershed algorithm to segment the medical images. Fuzzy C-Means clustering methods were integrated with Marker-controlled watershed segmentation to reduce the over segmentation problem. K-means clustering with watershed algorithm gave better results than Fuzzy Cmeans clustering.

Nirmala and Gowri (2013) proposed an enhanced FCM algorithm called Ameliorate FCM for lung CT image segmentation. The input images are classified into normal or abnormal images using hybrid feature selection method. The AFCM algorithm then clusters the pixels of the images based on its membership value to segment the region.

Lassen et al. (2013) proposed a lobe segmentation method based on fissure segmentation. The fissures were enhanced using eigen values of Hessian matrix and two features were derived from it. The strength of image structure and the discriminating factor with the other structures were calculated. These features gave the overall fissure similarity measure and it was used for lobe segmentation.

Shojaii et al. (2005) applied the marker-based watershed segmentation to automatically segment the lungs in their work. The method work by finding the internal and external markers and gradient image of the original data was combined with the markers. Watershed transform was then applied to find the lung borders. The method requires no prior anatomical knowledge and it is fully automatic.

From the Literature survey, it is evident that the major works can be categorized broadly into two based on the application of prior anatomical knowledge. The accommodation of the methods for variations in the individuals and their robustness varies. Our proposed method does not incorporate prior anatomical knowledge except the structure of fissures and the variations in the individuals is also accommodated.

\section{MATERIALS AND METHODS}

Watershed transformation is an effective tool for morphological image segmentation (Alina and Gabbouj, 1998). Two broad approaches in watershed construction are 'immersion based' and 'rainfall flooding'. The underlying idea takes the topographical nature of the region, a relief flooded by water, with the watershed lines dividing the catchment basins. The immersion based (Vincent and Soille, 1991) process starts with holes pierced called minima. The water starts immersing from the holes or minima and fills the basins. The flooding stops when all the basins are grown and to avoid merging of water from different basins, dams are constructed.

The end of the process results in catchment basins separated by watershed ridge lines giving the segmented region. The problem with the traditional method is that it results in over segmentation (Gonzalez and Woods, 2008). To overcome the problem, several approaches have been proposed like marker based watershed transformation.

In the current work, we propose an automatic method to segment the fissures based on 2D markerbased watershed transform. The method is completely automatic and no prior anatomical knowledge is required. It consists of several steps in obtaining the fissures segmented.

First, the gradient image of the original image is obtained. The internal and external objects are then marked to find the local minima. Watershed transformation is then applied to the image which has regional minima only in the specified marked positions. The result of the transformation is then superimposed on the original image to obtain the segments. As a post-processing step a novel method called SSRA is adopted to remove the small segments present along with the fissures. It results in the segmentation of the fissures alone. The architecture of the proposed method is as shown in Fig. 1.

\subsection{Preprocessing}

Sobel filter is applied as the first step to preprocess and obtain gradient magnitude of the image. The Sobel filter produce an approximated image gradient of the corresponding image. Resulting image contains gradient high at the borders and less gradient inside the object. The Sobel filter also smoothens the image to an extent by suppressing the noise present in the image. The following steps in the process require the edges to be enhanced to specify the internal and external markers. The preprocessing step results in an image with the edges enhanced enough to specify the markers. The original image and the gradient magnitude is as shown in the Fig. 2. 


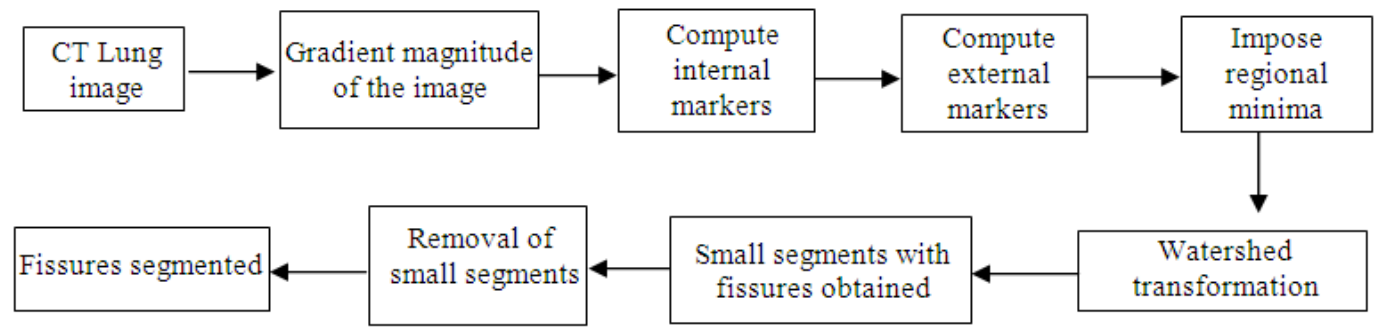

Fig. 1. Architecture diagram of the proposed method

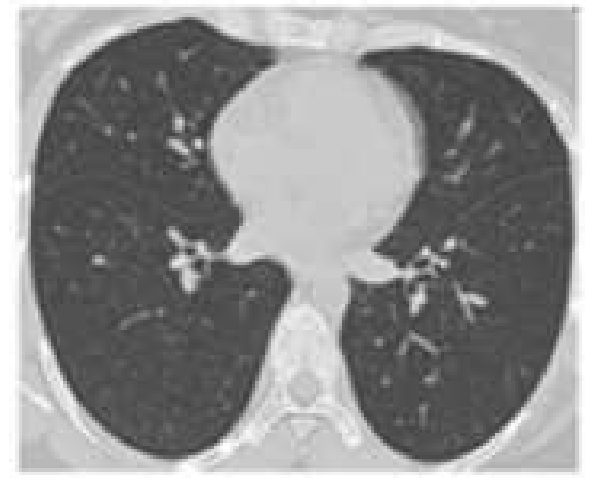

(a)

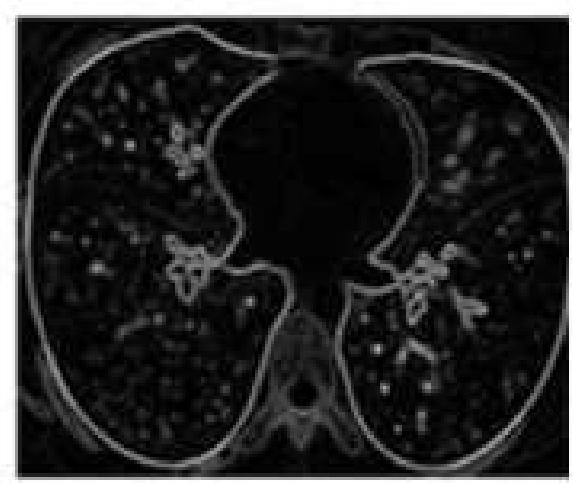

(b)

Fig. 2. (a) Original CT image (b) gradient magnitude of the image

\subsection{Internal Markers}

Over segmentation is a common problem in watershed transformation as all the regional minima forms a catchment basin resulting in uncountable basins. To avoid over segmentation, we specify the internal markers to construct the regional minima and grow basins around the marked minima regions. Markers are the connected components of the pixels within the object with an intensity value varying from the surrounding pixels. Morphological operations are applied to find the markers thatresults in flat maxima inside each of the objects. As a first step, an appropriate structuring element is chosen to perform the morphological opening on the grayscale image.

Erosion and Dilation are performed on the objects to obtain the maxima of the region. Image after applying the morphological operators is superimposed on the original image to identify the internal markers.

\subsection{External Markers}

The background markers are the dark pixels present in the image and to differentiate it from the foreground objects, a distance transform is applied. The foreground objects are separated from touching the edges of the background objects by applying a watershed transform and watershed ridgelines are obtained. The internal and external markers obtained are as shown in Fig. 3.

\subsection{Impose Regional Minima}

The flooding in a watershed basin starts from a regional minima and the watershed ridge lines are constructed. The regional minima in the specified foreground and background objects is found out by sorting the pixels in each of the marked objects and then the pixels with the minimum intensity is chosen. Watershed transformation is then applied on the marked object with the initial minimal point identified. The watershed starts flooding from the minima and the basins are constructed with watershed ridge lines between the regions.

\subsection{Post processing}

The results obtained after applying watershed transformation detects fissures along with accessory fissures and other small non fissure regions. 


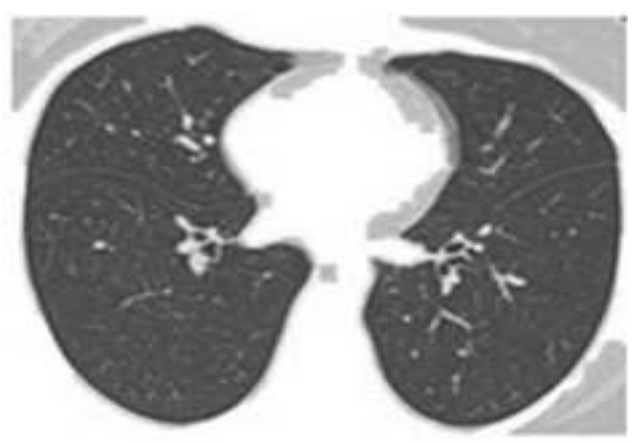

(a)

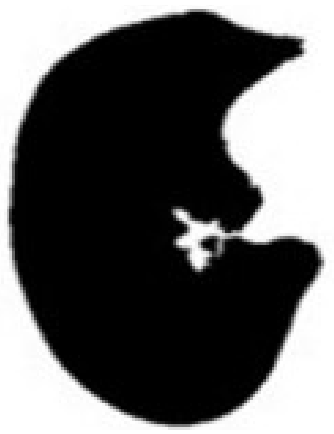

(b)

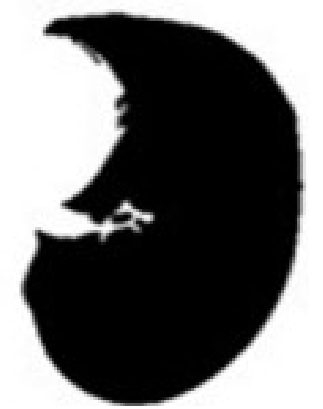

Fig. 3. (a) CT image with internal markers (b) CT image with external markers

A novel method called Small Segment Removal Algorithm (SSRA) is then applied to remove the accessory fissures and other non fissure smaller regions based on thresholding technique. Fissures are seen as the longest connected component of pixels with a plate like structure in the lung CT image. This unique feature of the surface structure of fissures is used in determining the thresholding factor of the SSRA algorithm. The thresholding factor is obtained as a normalized value of the length of the fissures of 20 sample lung CT images. Structures smaller than the determined threshold are then removed to retain the fissures alone.

The SSRA algorithm takes the gray image I as input and the output gray image f show the result with the fissures detected. The algorithm scans the object from the top left to right and the pixels are processed to retain the fissure structure alone. A queue is being initialized to process the scanned pixels. The pixels are processed in order and the gray value of the neighbouring pixels are compared.

The pixels with the same gray level value of the same connected component are input into the queue in order and the count of pixels is maintained. The count of pixels is compared with the predetermined threshold value after each pixel is added to the queue. When the count of pixels in the queue is below the predetermined threshold, after processing all the pixels in the same connected component, it is removed. The connected component with value larger than the threshold is retained, as it represents the fissures.

The SSRA algorithm detects the fissures based on its unique feature as the longest connected component. Accessory fissures and non fissure structures are eliminated as they are structures below the predetermined threshold.

\section{SSRA Algorithm}

Input: The input gray image I
Output: The output gray image with fissures segmented $f$ Initialization

P-current pixel

P'-Neighbouring pixel

q-Queue of pixels that gives the connected component $\mathrm{g}(\mathrm{P}), \mathrm{g}\left(\mathrm{P}^{\prime}\right)$-gray scale value of $\mathrm{P}$ and $\mathrm{P}^{\prime}$

t-threshold value

C-count of pixels

1. $\mathrm{C}=0 / /$ Initialize the counter to Zero

2. Rasterscan(I) // Start scanning the image

3. \{

4. $\mathrm{q} \leftarrow \mathrm{P} / /$ Enqueue the current pixel to the queue

5. do// Start adding the pixels of the same connected component to the queue

6. \{

7. $\left\{\right.$ If $\left(\left(\mathrm{g}(\mathrm{P})=\mathrm{g}\left(\mathrm{P}^{\prime}\right)\right)\right.$

9. Enqueue $(\mathrm{q}) \leftarrow \mathrm{P}^{\prime}$

10. $\mathrm{C}=\mathrm{C}+1$

11$.

12. else

13. $\{$ dequeue(q) $\} / /$ End of if

14. $\}$ while (end of queue(q)) // End of queue

15. if $(\mathrm{C}<\mathrm{t}) / /$ Check the length of the current connected component

16. \{

17. Delete(q)

18. $/ /$ End of if

19. else if $(C>=t)$

20. \{display (q) \} // Display the connected component of length larger than the threshold

21. free ( C) // Delete the contents of the counter variable 22.

23. End scan (I)

The results shown in Fig. 4a depicts the intermediate results with fissures and small segments. The fissures obtained after the preprocessing step with SSRA algorithm is as shown in Fig.4 b. 


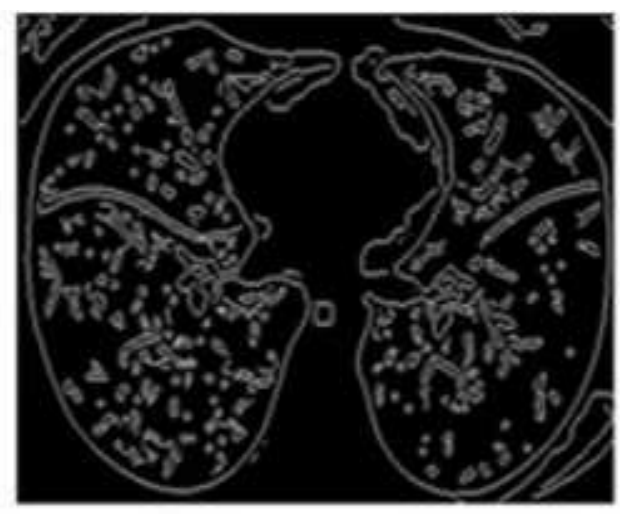

(a)

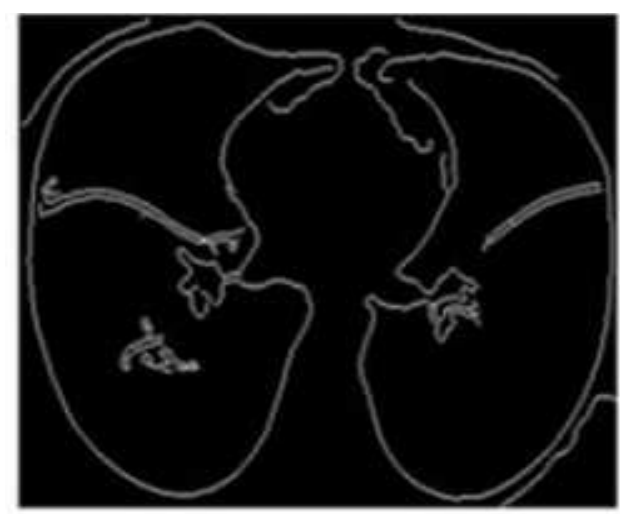

(b)

Fig. 4. (a) CT image with fissures and other smaller regions (b) CT image with fissures segmented

\subsection{Experimental Method}

The method was experimented with 65 lung CT images selected from $6 \mathrm{CT}$ examinations of both normal and diseased subjects. They were acquired under normal conditions with $140 \mathrm{Kv}, 250 \mathrm{~mA}$ and thickness of $1.2 \mathrm{~mm}$.

All the images are zrepresented using a $512 * 512$ matrix. The machine used for all the experiments was a 2.6 GHz Pentium IV system with a 3 GB RAM.

The performance of the method is validated by comparing the automatic segmentation results with a standard manually defined by an expert radiologist.

The expert radiologist manually traced the fissures for all the $65 \mathrm{CT}$ images and defined a standard. The dataset contains images with both complete and incomplete fissures.

The automated method proposed is capable of detecting only the visible fissure regions and hence the expert traced the fissures as a continuous curve to the extent where the fissures are visible. The expert observer marked points on the fissures to trace the curves and the points on the fissures detected by the automatic segmentation method is compared to match the points.

The validation of the performance is measured by the number of matching points. Error distance between the non matching points is measured using Eucledian distance calculator given by $\sqrt{\left(\mathrm{x}_{1}-\mathrm{x}_{2}\right)^{2}+\left(\mathrm{y}_{1}-\mathrm{y}_{2}\right)^{2}}$ where $\left(\mathrm{x}_{1}, \mathrm{y}_{1}\right)$ is the manually plotted point and $\left(\mathrm{x}_{2}, \mathrm{y}_{2}\right)$ is the point marked by the automatic segmentation method. The accuracy of the method is given in terms of the number of matching points, percentage of error and the error distance measured in $\mathrm{mm}$.

\section{RESULTS}

The results of the proposed method when tested with lung CT images are as shown in Fig. 5. Results obtained for a pathological lung CT image is as shown in the Fig. 6. Table 1 summarizes the results obtained after experimenting with 10 sample CT image for detecting left and right oblique fissures. The expert radiologist traced the fissures by plotting 150 points on the fissure surface. The proposed method is applied to segment the fissures and the number of matching points compared with the expert observation is recorded.

The performance of the method is assessed by calculating the error percentage obtained by comparing the number of matching points between the expert observation and the automated method. The error distance given in $\mathrm{mm}$ is measured using Eucledian distance calculator.

The average error percentage is seen to be 3.82 in the case of rigth oblique fissure and 3.96 for left oblique fissures. The maximum error distance is $1.365 \mathrm{~mm}$ and $1.502 \mathrm{~mm}$ for rigth and left oblique fissures respectively.

Table 2 summarizes the Average Error Distance (AED), RMS and SD for the left and right oblique fissures. It could be observed that the method produced better results for the rigth compared to left oblique fissure.

Figure 7 and 8 show the curves for the points plotted and the error percentage. From the curves, it could be observed that for the samples 4, 5, 6, 7 and 8 the number of mismatched points and error percentage is more when experimented to detect left oblique fissures.

Similarly, while segmenting right oblique fissures the samples 4, 5 and 6 show higher error percentages than other samples. Figure 9 shows the comparison of the results obtained for left and right oblique fissures. 
Devaki, K. and V. Murali Bhaskaran / Journal of Computer Science 10 (6): 896-905, 2014
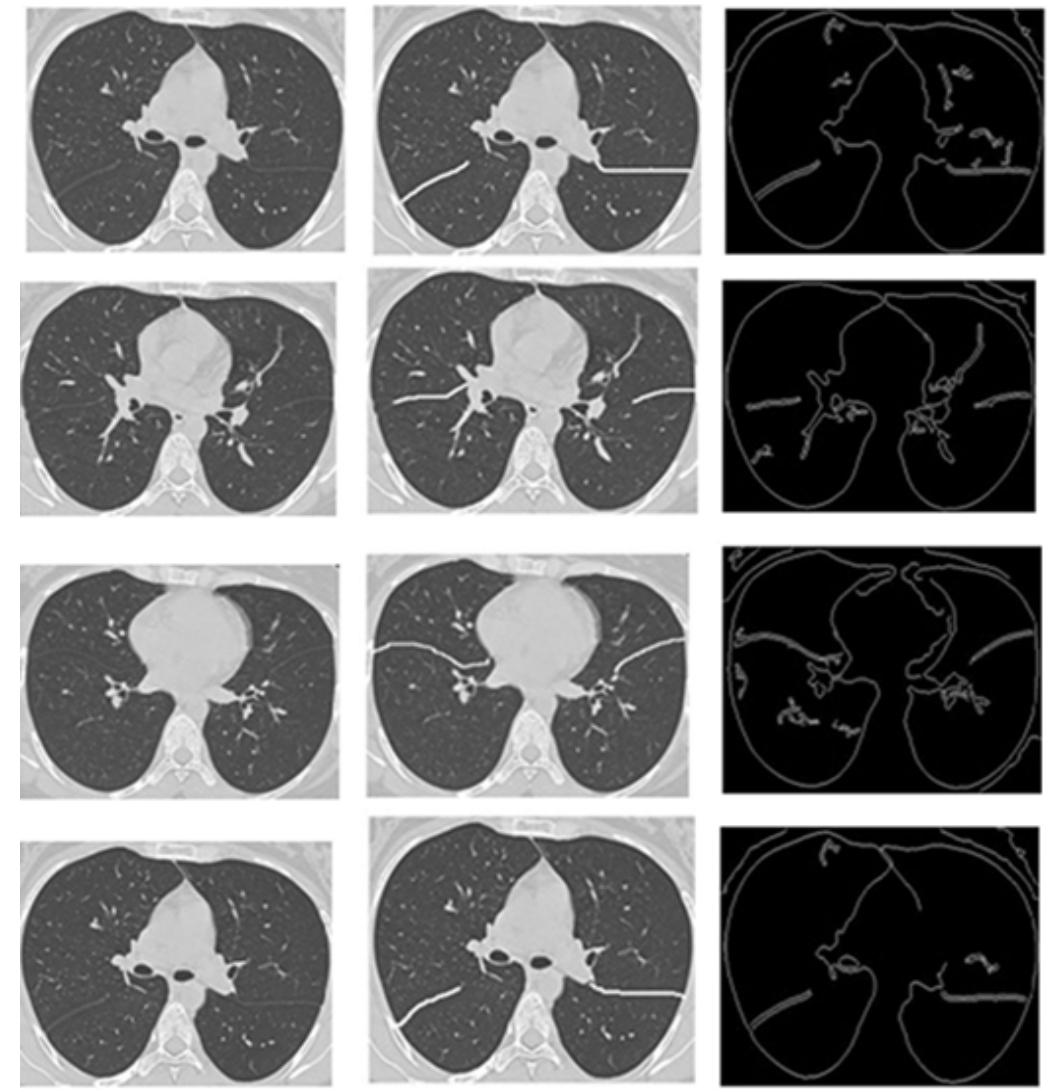

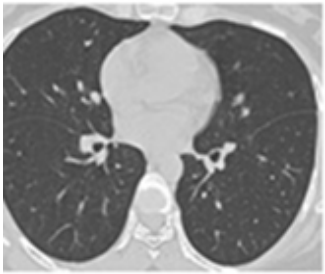

(a)

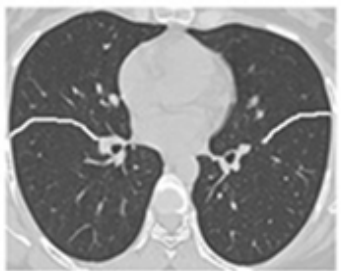

(b)

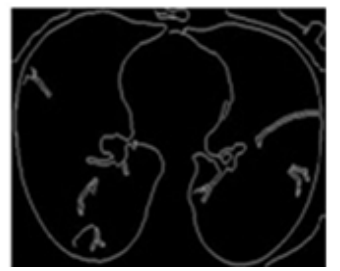

(c)

Fig. 5. Results of segmentation (a) original image (b) manual tracing (c) left and right oblique fissure segmentation

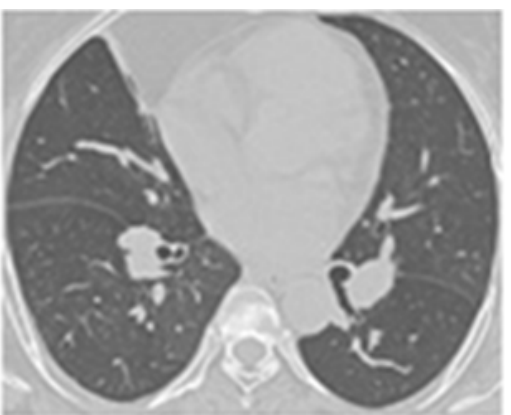

(a)

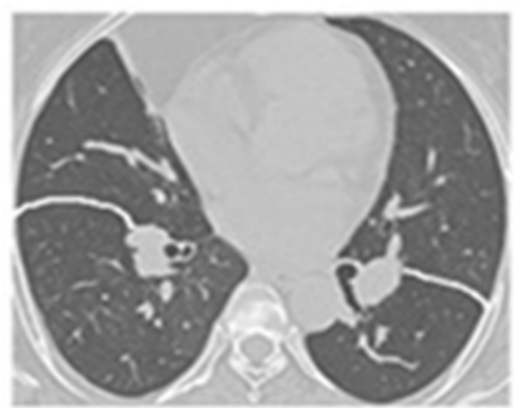

(b)

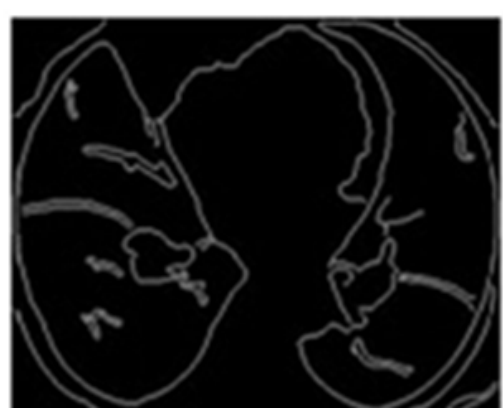

(c)

Fig. 6. Results of segmentation (a) Original pathological image (b) manual tracing (c) left and right oblique fissure segmentation 
Devaki, K. and V. Murali Bhaskaran / Journal of Computer Science 10 (6): 896-905, 2014

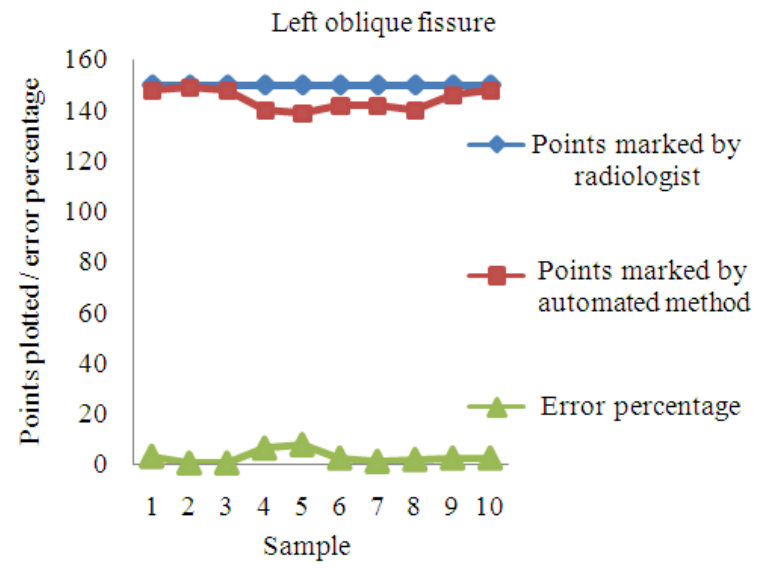

Fig. 7. Curves showing the matching points and error percentage for left oblique fissure segmentation

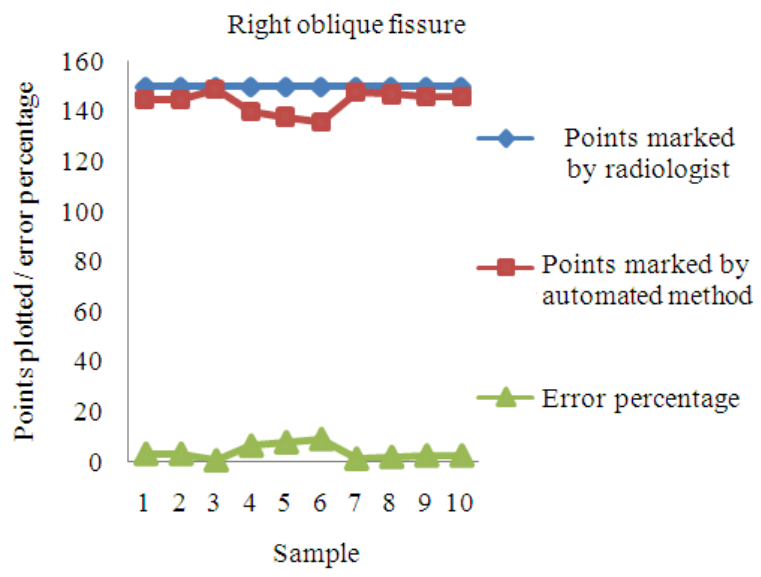

Fig. 8. Curves showing the matching points and error percentage for right oblique fissure segmentation

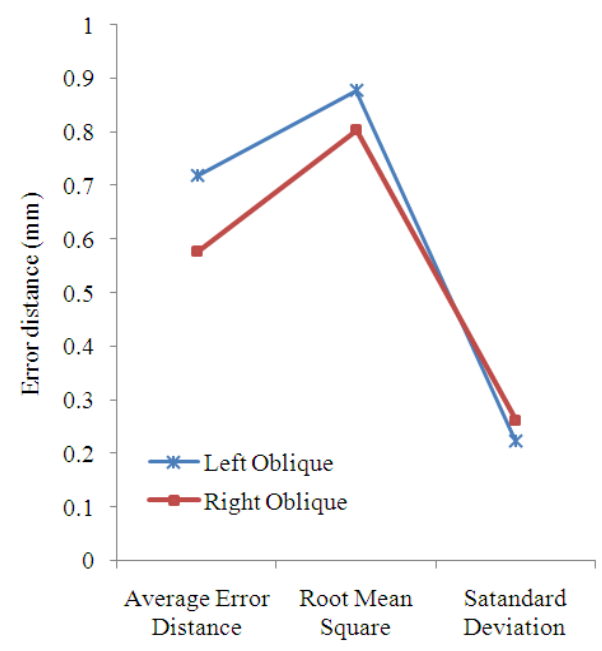

Fig. 9. Curves showing comparative AED, RMS and SD 
Table 1. Error measurement for left oblique fissure and right oblique fissure

\begin{tabular}{|c|c|c|c|c|c|c|c|c|}
\hline \multirow[b]{2}{*}{$\begin{array}{l}\text { Name of } \\
\text { the sample }\end{array}$} & \multicolumn{4}{|c|}{ Left oblique fissure } & \multicolumn{4}{|c|}{ Rigth oblique fissure } \\
\hline & $\begin{array}{l}\text { No. of } \\
\text { points marked } \\
\text { by radiologist }\end{array}$ & $\begin{array}{l}\text { No. of matching } \\
\text { points by } \\
\text { automated method }\end{array}$ & $\begin{array}{l}\text { Error } \\
(\%)\end{array}$ & $\begin{array}{l}\text { Error } \\
\text { distance } \\
(\mathrm{mm})\end{array}$ & $\begin{array}{l}\text { No. of } \\
\text { points marked } \\
\text { by radiologist }\end{array}$ & $\begin{array}{l}\text { No. of matching } \\
\text { points by } \\
\text { automated method }\end{array}$ & $\begin{array}{l}\text { Error } \\
(\%)\end{array}$ & $\begin{array}{l}\text { Error } \\
\text { distance } \\
(\mathrm{mm})\end{array}$ \\
\hline 1 & 150 & 148 & 1.3 & 0.137 & 150 & 145 & 3.3 & 0.203 \\
\hline 2 & 150 & 149 & 0.6 & 0.154 & 150 & 145 & 3.3 & 0.210 \\
\hline 3 & 150 & 148 & 1.3 & 0.327 & 150 & 149 & 0.6 & 0.152 \\
\hline 4 & 150 & 140 & 6.6 & 1.242 & 150 & 140 & 6.6 & 1.313 \\
\hline 5 & 150 & 139 & 7.3 & 1.365 & 150 & 138 & 8.0 & 1.465 \\
\hline 6 & 150 & 142 & 5.3 & 1.051 & 150 & 136 & 9.3 & 1.502 \\
\hline 7 & 150 & 142 & 5.3 & 1.043 & 150 & 148 & 1.3 & 0.261 \\
\hline 8 & 150 & 140 & 6.6 & 1.342 & 150 & 147 & 2.0 & 0.252 \\
\hline 9 & 150 & 146 & 2.6 & 0.215 & 150 & 146 & 2.6 & 0.203 \\
\hline 10 & 150 & 148 & 1.3 & 0.316 & 150 & 146 & 2.6 & 0.199 \\
\hline
\end{tabular}

Table 2. Comparative AED, RMS and SD measures for right oblique fissure and left oblique fissure

\begin{tabular}{lccc}
\hline Fissure type & AED & RMS & SD \\
\hline Left oblique & 0.7192 & 0.8772 & 0.2243 \\
Right oblique & 0.5760 & 0.8030 & 0.2620 \\
\hline
\end{tabular}

Table 3.

\begin{tabular}{llll}
\hline & RMS (mm) & & \\
& & Fast & Marker Based \\
Fissures & Watershed & watershed & watershed \\
\hline Right Oblique & 1.99 & 1.87 & 0.8030 \\
Left Oblique & 2.12 & 1.81 & 0.8772 \\
\hline
\end{tabular}

Table 3 summarises the comparative rms error for the methods employed with conventional watershed methods. The comparison shows that the method is more accurate than the traditional methods as it overcomes the over segmentation problem.

\section{DISCUSSION}

In the current method, we have proposed and tested an automatic method to detect and segment the fissures. The proposed method has many advantages and its limitation is trivial. Primarily, the method does not depend on the prior anatomical knowledge of the individual subjects. A direct automatic segmentation method is employed that do not require any information about the individual subjects and it paves way to reduce the dependence of the method on the variations of the anatomy. The fact that the fissures are the long connected component of pixels with a plate like structure is taken into consideration. This assumption helps in detecting the fissures eliminating other non fissure surfaces and fissure like plates.

Further, fissures in pathological lung dataset can also be detected and segmented using the proposed method. As only the visible surface of the fissures is detected and segmented, the method could well be deployed to detect incomplete fissures and fissures in pathological data set also.

A trivial limitation of the current method is that, plate like structures in pathological lung images may be misclassified as fissures due to its shape appearance. Incomplete fissures below the determined threshold may be wrongly eliminated as small structures using the current method.

Absence of a standard dataset to evaluate the performance remains another limitation. The assessment of the performance of the proposed method lies on the standard defined by the expert radiologist alone. The results obtained are promising and reliable compared to the expert observation and lies in an acceptable range.

\section{CONCLUSION}

We have developed a new automated method to detect and segment the pulmonary fissures in lung CT images. It employs marker based watershed transformation to detect the fissures and a novel approach called SSRA is applied as a post processing step to remove the non fissure and fissure like plate structures to segment the fissures alone.

The method does not depend on the prior anatomical knowledge of the individual subjects. The unique plate like structure of the fissures as a long connected component is considered to detect the fissures. The performance of the 
method is evaluated in comparison with an expert radiologist observation. The rms error ranges from $0.877 \pm 0.224 \mathrm{~mm}$ for left oblique fissure and $0.803 \pm 0.262$ $\mathrm{mm}$ for right oblique fissure and the accuracy of the method is found to be $96.61 \%$ compared to the human observer. The method is found to be fast, accurate and reliable.

\section{ACKNOWLEDGEMENT}

The researchers would like to thank Dr. P. Senthil Kumar, Consultant Radiologist, Saravana Scans, Salem, India for providing valuable suggestions and results of manual tracing, Dr.R. Saravanan, Director, Deepam Hospitals, Chennai, India for the data set used in this study.

\section{REFERENCES}

Alina, N.M. and M. Gabbouj, 1998. Parallel markerbased image segmentation with watershed transformation. J. Parallel Distributed Comput., 51: 27-45. DOI: 10.1006/jpdc.1998.1448

Christ, M.C.J. and R.M.S. Parvathi, 2011. Segmentation of medical image using clustering and watershed algorithms. Am. J. Applied Sci., 8: 1349-1352. DOI: 10.3844/ajassp.2011.1349.1352

Gomathi, M. and P. Thangaraj, 2010. A computer aided diagnosis system for lung cancer detection using support vector machine. Am. J. Applied Sci., 7: 15321538.DOI: 10.3844/ajassp.2010.1532.1538

Gonzalez, R.C. and R.E. Woods, 2008. Digital Image Processing. 3rd Edn. Prentice Hall of India.

Jiantao, P., B. Zheng, J.K. Leader, C. Fuhrman and F. Knollmann et al., 2009. pulmonary lobe segmentation in ct examinations using implicit surface fitting. IEEE Trans. Med Imag., 28: 19861996. DOI: 10.1109/TMI.2009.2027117

Jingbin, W., M. Betke and P.K. Jane, 2006. Pulmonary fissure segmentation on CT. Med. Image Anal. DOI: 10.1016/j.media.2006.05.003

Lassen, B.E. M. Jan-Rikxoort, M. Schmidt, S. Kerskta and B. Van Ginneken et al., 2013. Automatic segmentation of the pulmonary lobes from chest CT scans based on fissures. IEEE Trans. Med Imag. Vessels Bronchi., 32: 210-222. DOI: 10.1109/TMI.2012.2219881

Lin-Yu, T. and H. Li-Chin, 2010. Automatic fissure detection in CT images based on genetic algorithm. Proceedings of the 9th International Conference on Machine Learning and Cybernetics, Jul. 11-14. IEEE Xplore Press, Qingdao, pp: 2583-2588. DOI: 10.1109/ICMLC.2010.5580871
Nirmala, B.J. and S. Gowri, 2013. Ameliorate fuzzy cmeans: An ameliorate fuzzy c-means clustering algorithm for ct-lung image segmentation. Am. J. Applied Sci., 10: 1439-1447. DOI: 10.3844/ajassp.2013.1439.1447

Ochs, R.A., J.G. Goldin, F. Abtin, H.J. Kim and K. Brown et al., 2007. Automated classification of lung bronchovascular anatomy in CT using AdaBoost. Med. Image Anal., 11: 315-324. DOI: 10.1016/j.media.2007.03.004

Qiao, W., Y. Hu, J.H. MacGregor and G. Gelfand, 2008. Segmentation of lung lobes in clinical CT images. Int. J. CARS. 3: 151-163. DOI: 10.1007/s11548008-0153-5

Rikxoort, E.M.V., D.H. Bartjan, V.D.V. Saskia, M. Prokop and V.G. Bram, 2009. Automatic segmantation of pulmonary segments from volumetric chest ct scans. IEEE Trans. Med. Imag., 28: 621-630.2009. DOI: 10.1109/TMI.2008. 2008968

Ross, J.C., R. San J. Washko, A. Diaz and C.F. Westin et al., 2009. Lung extraction, lobe segmentation and hierarchial region assessment for quantitative analysis on high resolution computed tomography images. Proceeding of the 13th International Conference on Medical Image Computing and Computer-Assisted Intervention, pp: 690-98. DOI: 10.1007/978-3-642-04271-3-84

Ross, J.C., R. San J. Washko, A. Diaz and C.F. Westin et al., 2010. Lung lobe segmentation using particles, thin plate splines and maximum a posteriori estimation. Proceeding of the 14th International Conference on Medical Image Computing and Computer-Assisted Intervention, Springer, New York, pp: 163-171. DOI: 10.1007/978-3-642-15711$0 \_21$

Shojaii, R., J. Alirezaie and P. Babyn, 2005. Automatic Lung Segmentation in CT Images using Watershed Transform. Proceedings of the IEEE International Conference on Image Processing, Sep, 11-14. IEEE Xplore Press, pp: 1270-1273. DOI: 10.1109/ICIP.2005.1530294

Soumik, U. and J.M. Reinhardt, 2009. Anatomy-guided lung lobe segmentation in $\mathrm{x}$-ray $\mathrm{CT}$ images. IEEE Trans. Med. Imag., 28: 202-214. DOI: 10.1109/TMI.2008.929101

Vincent, L. and P. Soille, 1991. watersheds in digital spaces: An efficient algorithm based on immersion simulations. IEEE Trans. Patt. Anal. Machine Intell., 13: 583-598. DOI: 10.1109/34.87344

Zhang, L., E.A. Hoffman and M.R. Joseph, 2006. Atlasdriven lung lobe segmentation in volumetric $\mathrm{X}$-ray CT Images. IEEE Trans. Med. Imag., 25: 1-16. DOI: 10.1109/TMI.2005.859209 